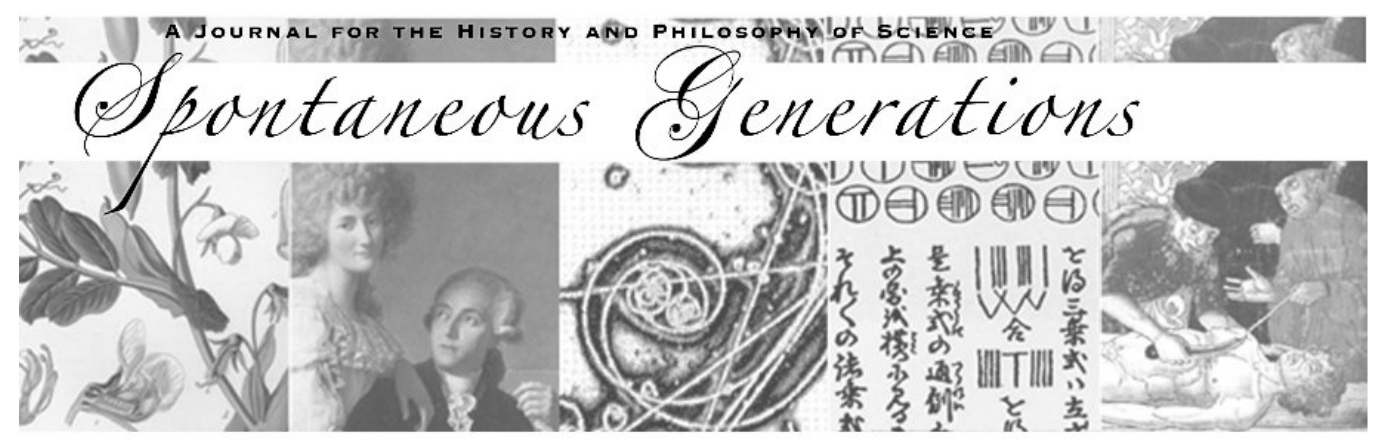

\title{
Extended Thing Knowledge
}

Author(s): Mathieu Charbonneau

Source: Spontaneous Generations: A Journal for the History and Philosophy of Science, Vol. 4, No. 1 (2010) 116-128.

Published by: The University of Toronto

DOI: $10.4245 /$ sponge.v4i1.11891

EDITORIAL OFFICES

Institute for the History and Philosophy of Science and Technology Room 316 Victoria College, 91 Charles Street West

Toronto, Ontario, Canada M5S 1K7

hapsat.society@utoronto.ca

Published online at jps.library.utoronto.ca/index.php/SpontaneousGenerations ISSN 19130465

Founded in 2006, Spontaneous Generations is an online academic journal published by graduate students at the Institute for the History and Philosophy of Science and Technology, University of Toronto. There is no subscription or membership fee. Spontaneous Generations provides immediate open access to its content on the principle that making research freely available to the public supports a greater global exchange of knowledge. 


\title{
Extended Thing Knowledge ${ }^{*, \dagger}$
}

\author{
Mathieu Charbonneau ${ }^{\ddagger}$
}

This paper aims at extending the notion of thing knowledge put forth by Davis Baird. His Thing Knowledge (Baird 2004) proposes that scientific instruments constitute scientific knowledge and that to conceive scientific instruments as such brings about a new and better understanding of scientific development. By insisting on what "truth does for us," Baird shows that the functional properties of truth are shared by the common scientific instrument. The traditional definition of knowledge as justified true belief would only apply to scientific instrumentation if we were to reject the subjective (and intentional) aspect of it, viz. belief. In consequence, Baird insists that scientific instruments can be seen only as a kind of objective knowledge, that is knowledge maintained by the scientific community but not individually by a given scientist. In this paper, I will argue for a conception of subjective thing knowledge according to which some scientific instruments (especially material models) can be understood as justified true beliefs. By combining Davis Baird's thing knowledge and Clark and Chalmers' hypothesis of extended cognition, I will show that it is possible to derive an analysis of material models as cognitive augmentation of the scientist's mind, and that such scientific instruments are to be understood as a material form of subjective knowledge, that is, as external-to-the-brain justified true beliefs.

Material models do not give rise to any ontological difficulties over and above the well-known quibbles in connection with objects, which metaphysicians deal with.

Frigg and Hartmann 2006

This paper aims at extending the notion of thing knowledge put forth by Davis Baird. His Thing Knowledge (Baird 2004) proposes

* I would like to thank Jean-Pierre Marquis for his helpful comments on earlier versions of this paper and for his support without which this paper would never have seen the light of day. I would also like to thank Guillaume Beaulac and the two anonymous referees for their useful comments. The research behind this paper was funded by le Fond québécois de la recherche sur la société et la culture.

$\dagger$ Received 21 February 2010. Revised paper accepted 15 April 2010.

$\ddagger$ I am a PhD Candidate at the Department of Philosophy at the Université de Montréal. My research interests in the philosophy of science include evolutionary biology, environmental sciences, cognitive science, and evolutionary anthropology. 
that scientific instruments constitute scientific knowledge and that to conceive scientific instruments as such brings about a new and better understanding of scientific development. Using Popper's conception of objective knowledge, Baird shows that a pragmatic conception of truth and justification allows one to talk about scientific instrumentation as public scientific knowledge. By insisting on what "truth does for us," Baird shows that the functional properties of truth are shared by the common scientific instrument. Although the idea of knowledge in a non-declarative and material form seems counter-intuitive, Baird argues that to conceive scientific instruments as such helps us better understand the ongoing work of scientific communities. Furthermore, Baird insists that in many historical instances of scientific development, the instruments used in the field of inquiry played a justificatory and explanatory role that is usually occupied by declarative scientific theories.

The traditional definition of knowledge as justified true belief would only apply to scientific instrumentation if we were to reject the subjective (and intentional) aspect of it, viz. belief. In consequence, Baird insists that scientific instruments can be seen only as a kind of objective knowledge, that is knowledge maintained by the scientific community but not individually by a given scientist.

In this paper, I will argue for a conception of subjective thing knowledge according to which some scientific instruments (especially material models) can be understood as justified true beliefs. This is not done in order to argue against Baird's account of objective thing knowledge, but to complete it. Since each conception complements the other I will not contrast subjective and objective thing knowledge. This is the main reason why I label my view as "extended" thing knowledge.

The other reason for this label is that my claim for subjective thing knowledge will be made by invoking the extended cognition perspective introduced by Andy Clark and David Chalmers (Clark and Chalmers 1998) according to which a cognitive system is not restricted to someone's brain but can extend into the world if some part of the world plays the proper cognitive role for that system. By combining Davis Baird's thing knowledge and Clark and Chalmers' hypothesis of extended cognition, I will show that it is possible to derive an analysis of material models as a cognitive augmentation of the scientist's mind, and hence that such scientific instruments are to be understood as a material form of subjective knowledge. Although I argue for actual cases of such external-to-the-brain justified true beliefs elsewhere, I will restrict myself here to demonstrating the mere possibility of such extended thing knowledge. To illustrate this possibility I will offer a hypothetical example that illustrates a case of such materialized subjective knowledge. 
The paper will be organized as follows. I first offer a brief discussion of Baird's 'thing knowledge' thesis and comment on the need for a subjective account of such materialized knowledge. The following section will serve as a brief exposition of the hypothesis of extended cognition and will flesh out a common assumption that both the thing knowledge and the extended cognition theses share: they both endorse functionalism and the multiple realizability principle. I will then turn to a characterization of material beliefs and will offer an example of a use of material models that complies with a functional account of knowledge. It is important to bear in mind that I will not argue for either the thing knowledge or extended cognition theses. I do however hope that my view will support both theses and show that they should be included in the epistemologist's tool kit.

\section{Objective Thing KnOWLedge}

Baird argues that considering scientific instruments as genuine knowledge will enhance our understanding of science in historical and philosophical perspectives. Although Baird argues that different kinds of scientific instruments exhibit many different forms of knowledge, I will restrict my attention here to one kind of scientific instrument, viz. material models. According to this view, a material model representing some natural system (e.g. a given molecule such as the $\alpha$-keratin protein) should be understood in basically the same way as its linguistic counterpart (a theory about the structure of such protein): they both are representations on the basis of which scientific explanations and predictions can be made.

Baird's conception of scientific instruments as knowledge rests on a functional account of knowledge, by the use of what he terms a "thin conception of function" (Baird 2004, 123-25). By offering an answer to the question "what does knowledge do for us?" (Baird 2004, 119), where "us" refers to the scientific community as a whole, Baird offers an account of the role of truth and justification of scientific knowledge. It is because scientific instruments instantiate such functional roles for the scientific community (just like theories do) that they can be considered not only as analogous to knowledge but as knowledge per se.

According to Baird (2004, chapter 6), material models, like true theories, exhibit detachment (they are multi-contextually applicable), efficacy and longevity (we can depend on them now and into the indefinite future), connection (they establish a relation between us and the world) and objectivity (the world has priority over their working, not our minds or our wishful thinking). Together, these five functions constitute the function of truth, or what Baird terms "material truth" (Baird 2004, 119-23). ${ }^{1}$ Like

${ }^{1}$ Although it is beyond the aim of this paper to argue for such a functional conception of 
theories being justified because they cohere and are supported by other previously accepted theories, instruments are justified because their truth (understood in terms of the five ideals presented above) is supported by an array of previously reliable instruments that were used in their making. They are calibrated and built with the use of proven tools and approved scientific instruments and construction methods in the same way theories are built with sound logic and mathematics and previously approved scientific theories and methods.

According to Baird, material models comply with such requirements and should thus be understood as knowledge. It should be noted that Baird's thin conception of function rejects the mental (intentional) aspect of declarative knowledge. Baird is clear on this point: for him, whatever material models may be, they are not beliefs-they simply are not in someone's head (Baird 2004, 118). Material models implement the role of truth and justification but since they cannot be mental they do so for the scientific community as a whole: they are an objective kind of knowledge.

Baird shows that this understanding of material models as genuine knowledge, even if restricted to objective knowledge, can be very fruitful. But his claim is certainly a bold one, and one could well be confused by it at this point: for isn't representational knowledge usually understood as some kind of mental state about some part of the world? Indeed, once the mental (intentional) part of knowledge has been rejected, one is left with several questions about such objective thing knowledge. How does the community as a whole connect itself with the world on the basis of this knowledge? How is the content of material knowledge accessed and used by individual scientists when they aim to demonstrate or explain some fact about the world? There is certainly always some individual or group of individuals who built and used such models as representing a part of reality, but this is not the scientific community as a whole. Even Popper's criterion of objective knowledge, upon which Baird relies $(2002,26)$, seems to require a form of subjective use of objective knowledge:

In order to belong to the third world of objective knowledge, a book should-in principle, or virtually-be capable of being grasped (or deciphered, or understood, or 'known') by somebody. (Popper 1972, 116, my emphasis)

truth, a few comments may be in order. Baird's account of truth is pragmatic. In his 2002 paper, Baird elaborates these five ideals in order to allow one to conceive of a scientific instrument as being true although it is not representational (e.g. a cloud chamber). These five ideals also stand for representational forms of knowledge as illustrated by Baird's use of an encyclopaedic entry about plutonium (Baird 2002, 17). 
For the remainder of this paper I will argue that material models-the bearers of objective thing knowledge-can also be instances of subjective thing knowledge if they are used appropriately. Material models should not be considered as only public objects, and hence as in contrast with private mental states. Rather, we should consider them as part of an individual's cognitive states, if used in the right way. The task is then to show how it is that we can understand such subjective use of thing knowledge. My strategy will be to argue that material models can be construed as material beliefs by showing how a functional conception of belief can be implemented in material artefacts (outside the scientist's brain). This is where the hypothesis of extended cognition comes into play.

\section{EXTENDING THING KNOWLEDGE}

According to a functional view of the world (Lewis 1970; Shoemaker 1981), a type (i.e. any general sort of thing) is defined as a systematic causal relationship linking some states (inputs) to other states (outputs). In the case of declarative knowledge (as opposed to procedural knowledge), a type is assumed to relate trustworthy orderly inputs (sensory inputs and already known theories) to outputs (action instructions or actions per se).

The same can be said for those scientific instruments that model some part of the world. Because scientific knowledge-understood here as a type-is defined by its functional structure, whatever falls under the definition of the type is to be understood as knowledge. Given that material models meet this definition then according to the functional view they should be classed as knowledge. Whether it is scientific theories or scientific instruments that implement such a function, if the entity can be subsumed under the criteria of subjective knowledge then it has to be conceived of as a token of subjective knowledge. The material substratum is not important; only the functional implementation by a given entity matters.

The previous line of argumentation is very similar to the one offered by Clark and Chalmers (1998). According to the hypothesis of extended cognition, cognitive processes can extend outside the skull if some part of the world implements a cognitive role for the individual's cognitive system. To argue for their counterintuitive claim, Clark and Chalmers offer what they termed the "parity principle," which goes like this:

If, as we confront some task, a part of the world functions as a process which, were it done in the head, we would have no hesitation in recognizing as part of the cognitive process, then that part of the world is (so we claim) part of the cognitive process. (Clark 2008, 222) 
A now longstanding tradition in the philosophy of mind and cognitive science holds that mental states and cognitive processes are best understood as functions between sensory inputs and behavioural outputs (actions). According to this "functionalist" view, what qualifies something for being cognitive is the role it plays in the general economy of a cognitive system, not its actual materialization. This idea is known as the "multiple realizability principle": a cognitive state or process can be implemented in different material substrata. One can understand the parity principle as a reformulation of the consequences of adopting functionalism and the multiple realizability theses: it is of no consequence whether the cognitive system's actual material realization is of one type (neurons) or many (neurons + external-to-the-brain-stuff). What actually matters are the abstract cognitive roles playing a part in an individual's cognitive system. To put it differently, what matters is not the actor so much as the role being played. If this view is correct, extended cognition is a possibility that should be investigated by any cognitive account of science (e.g. see Carruthers et al. 2002).

Clark and Chalmers give an example of what should count as an "extended belief," viz. a belief that is not embedded in one's brain but in an external artefact. It is the case of Otto, a victim of Alzheimer's disease, who wants to go and visit MoMA. ${ }^{2}$ Otto does not have any internal belief about the museum's location. Instead, he uses the information about the museum address inscribed into a notebook he carries around with him. He then acts upon this information and, in conjunction with his desire to go to the museum, successfully reaches his destination. Now consider Otto's counterpart, Inga, for whom the information about the museum's location is encoded into her brain. In conjunction with her desire to go to the museum, Inga accesses her internal representation of the museum's address and acts upon it, reaching her destination successfully. In this example, Otto's use of the information inscribed into the notebook (about the museum's location) mirrors the role of Inga's internal belief of that same location. According to the parity principle stated above, should we recognize that Inga's internal representation of the museum's address plays the functional role of a belief, we ought to say that the external notebook information is also a belief instance since it plays the same functional role for Otto.

This line of reasoning allows for a conception of external-to-the-brain

2 Note that Otto's memory problem is not a necessary condition for the use of extended beliefs: in this example, the disease's role is to emphasize Otto's use of the notebook in accomplishing his task without any internal representation of the museum's location. Should Otto not have Alzheimer's disease and used the notebook in the same fashion (i.e., without relying on an internal representation), it would also count as a case of extended belief. 
beliefs-which I will call "material beliefs"-that can serve to complete Baird's account of thing knowledge. By showing that a material artefact can implement the cognitive role of a belief for a given scientist while also fulfilling the functional roles of truth and justification given above, one can lay claim to a genuine case of justified true beliefs and hence a case of subjective thing knowledge.

\section{MATERIAL BELIEFS}

In order to extend Baird's conception of thing knowledge to make it able to handle the subjective aspect of knowledge, one needs to answer to the question: What does belief do for us? In other words, what is the cognitive/functional role of a belief? This is no place to argue for a fine-grained conception of beliefs, and luckily this is not necessary for my view. Although thinking of material beliefs is counterintuitive, since beliefs are usually understood as internal cognitive states, the functional view to be developed below relies on our intuitions not about the locus of belief but instead on the role beliefs play in someone's cognitive economy. ${ }^{3}$ These intuitions are general enough to allow for a coarse-grained conception (that might seem to many to be a list of platitudes):

1. Beliefs are representational. Although beliefs are said to be some kind of propositional attitude, this seems to be too restrictive. Many of my mental representations are non-propositional although they serve the same functional role as some propositions we would have no hesitation to call beliefs. Non-propositional mental representations can play the same functional role we ascribe to propositional beliefs. The logical form of my representation need not be couched in a propositional format; what is required for it to represent some part of reality is that it is about that part of reality.

2. Beliefs allow for action. This point is crucial: a representation is a belief because it is used as such. Beliefs are used in accord with other beliefs and desires so that if one desires $X$ and believes that by doing $Y$ one will obtain $X$, then one will do $Y$. I understand action here as either behavioural actions or intellectual actions (such as mental image rotation or mental inferences, etc.).

3. Beliefs that are true lead to success. (Here truth is to be understood as it was defined above.) A belief that is true and used appropriately (e.g. in the correct context) will yield successful results when acted upon. In the case of scientific beliefs, one form of success in their use

3 See Clark $(2008,105-06)$ and Sprevak (2009) on this point. 
might be their production of adequate predictions, demonstrations or explanations. The kind of connection between the belief and the part of the world it aims at is also important in defining the success of an action based on such a belief. A true representation connects us with the part of reality it is about by sharing with it a structure similar in relevant ways. How the success and relevance of similarity should be understood is dependent on the task the belief user aims to solve. This rather abstract point will be illustrated more concretely in the next section.

4. Beliefs are recalled. ${ }^{4}$ Representations are not always actively used as beliefs; in some cases they must be recalled to one's memory for them to be acted upon. I will not attempt to define precisely what memory is; let us just say that in a very general sense, memory is some kind of representation storage.

These four conditions will have to be satisfied by a material artefact if one is to claim that it is a belief. If one does identify such an abstract functional role played by a material artefact when a given cognitive system uses it, then one can claim that it is a genuine belief. ${ }^{5}$ Note that in my view, for a representation to be a belief it must be used by someone according to the four properties above, that is, it must actually play the role of belief. Call this an active belief account. This implies that dispositional beliefs are not included in this account (while they remain compatible with it). The reason is simple, as Sprevak (2009) argues: anything that could play the role of belief, when accessed, would then be a belief. My picking up a book does not make the content of the book part of my active beliefs: I must use the content as such in order for it to count as belief (condition 2). ${ }^{6}$

Finally, if such materialized beliefs also implement the functional role ascribed to truth and justification, then one ought to talk about materialized knowledge-that is, subjective knowledge outside one's brain. Let us now see how material models can play such roles.

4 This feature of belief does not entail any specific degree of reliable access. It is sufficient that the representation can be accessed and that it is used according to the three previous conditions.

${ }^{5}$ Clark and Chalmers (1998) give an example of some materialized belief in their example of Otto wanting to go to the museum. (Clark 2008, 226-28)

6 This is clearly stated by Clark and Chalmers' (1998) parity principle (see above). It is intriguing that Sprevak in his criticism of the extended cognition does not take into account this well-known aspect of functions: something functions as an $\mathrm{X}$ if it is actually used as such. Mere possession of something that could play a given role does not make this thing an actual role-bearer. 


\section{MATERIAL MODELS AS MATERIAL BELIEFS}

To illustrate the possibility of subjective thing knowledge, I will offer a fictional case of a use of an orrery-a mechanical model depicting the motions of the Earth and the Moon relative to the Sun-which complies with the functional account of knowledge given above. The reasons why I will not use an actual or historical case are twofold. Firstly, any account of such an actual use of material models cannot be made without a detailed investigation of the representational properties of the models, of the actual use of these models by scientists and of the background theories in which they are embedded. Secondly, it is an empirical claim that any such use of material models complies with an account of subjective thing knowledge. Since I have set as my aim here to argue for the principled possibility of a subjective account of thing knowledge, I will not risk choosing a case that, after careful study, might not comply. I believe that the use of the orrery is simple enough to make my point but the reader should be warned that applying the concept of subjective thing knowledge is far from being as straightforward as it might seem on the basis of the following example (for the reasons mentioned above).

Let us imagine a scientist who wants to predict, given the actual state of the solar system, the date of the next full moon on Earth. The scientist knows (he can recall in his brain memory) the theory concerning the trajectories of the Earth and the Moon around the Sun. This scientist could use the relative positions of the Earth and Moon at time t, and with the proper use of the right mathematical formulas (known as astronomical algorithms), he will be able to calculate and thus predict their relative position at the time of the next full moon (when the ecliptic longitude of the Earth and Moon will differ by $180^{\circ}$ (e.g. see Meeus 1999). One is entitled to say that the scientist knows how this part of the solar system evolves through time and when the next full moon will occur: he subjectively knows a true astronomical theory on the basis of which he made correct predictions.

Now let us imagine another scientist with the same problem. Although this scientist is not in possession of an internal representation of the relevant formulas, he owns an orrery that adequately represents the relative motions of the Earth and Moon. An orrery is a mechanical device that represents the whole or part of the solar system and whose clockwork mechanism is able to represent the trajectory and relative motions of the astronomical bodies of our Solar System (in our example, those of the Earth and Moon relative to the Sun). This scientist does not "internally" know, as the first one does, the proper trajectory formulas or the theory to make the proper calculations. All that the second scientist internally knows is how to use an orrery to make the correct predictions, given that the 
orrery is calibrated adequately.

This second scientist approaches the problem as follows. ${ }^{7}$ He takes out his orrery from a drawer and puts it on his working desk. Given a description of the relative positions of the Earth and Moon at time t, he can set the starting positions of the different bodies on the orrery. By manipulating the material model, he can predict when the Earth and Moon will be positioned relatively to the Sun such as to cause a full moon. The material model offers the same predictions as the internalized astronomical theory, and hence its predictions are as accurate as those obtained in the first case. ${ }^{8}$

In this example, the orrery has played the same functional role for the second scientist that the astronomical bodies' relative position and trajectory formulas did for the first. All four conditions of beliefs are present, and so are those of truth and justification. The model is a representation of the astronomical bodies' trajectories and relative motion (condition 1). In conjunction with other beliefs (the initial positions of the Earth and Moon and the methodological guidelines for the manipulation of the orrery) and desires (wanting to know when the next full moon will be), the orrery allowed for actions. By setting the orrery to a state that was adequately representing the relative positions of the bodies at time $t$, and by using the orrery according to its proper manipulation guidelines, the second scientist was able to calculate the trajectories of the Earth and Moon until they reached the sought-for state. Instead of making calculations or using astronomical tables, the scientist relied on the proper manipulation of the orrery to do the computational job the first scientist did by calculating in his head (or on paper), allowing him to make the correct predictions. These actions (the manipulations and predictions) were successful (conditions 2 and 3), that is, as successful as the use of propositional theories and calculations were. Although the orrery does not represent astronomical bodies per se (the bodies' models on an orrery are out of scale), the relevant information about their relative position and motion is embedded in the model's material structure. This is the relevant representational structure of the orrery leading to scientific success in this case: the relative trajectories of the modeled bodies mirror the trajectories of the actual astronomical bodies. Moreover, in the first case the scientist had to recall from his internal memory the correct theories of motion and the correct method of calculating the trajectories. In the second case the

7 This thought experiment is an adaptation of an actual calculation made using an orrery by James Ferguson (Baird 2004, 25-29).

8 This accuracy is limited by the orrery's accuracy. This does not change the way the scientist uses the model (which is what is relevant here), for the same applies to the calculations of the first scientist. 
representational content was embedded in the orrery and was accessed when the material model taken out of the drawer and put to use (condition 4). The working desk on which the orrery was used played the role of a working memory, allowing the scientist to manipulate the model and make the correct predictions.

A final point must be made about the use of the orrery as a belief. After the orrery was put back into the drawer, or when the scientist left his office for a tennis game, the orrery stopped playing the role of a belief. If the scientist used the orrery to retain the piece of paper on which he made calculations of the trajectories because his office window lets in a strong breeze, the orrery was not playing the role of a belief. A material artefact is a belief only if it is used as such, and thus its status as a belief is merely temporary. As long and only as long as it is used as such, it plays the role of a belief.

The orrery also displayed the functional properties of truth and justification (since it was a correctly calibrated orrery). Recall the five ideals of truth. The model showed detachment (the scientist could have used the model for different similar calculations in other contexts), efficacy and longevity (the scientist could depend now or later on the model to make predictions) and connection (the orrery is a representation of relative motions and trajectories of the solar system's planets and moons). The last ideal of the functional account of truth, objectivity, also applies to the model. Suppose the second scientist did not use an orrery based on astronomical observations but one that was built according to his own preferences on what the correct relative motions of the astronomical bodies should be (e.g. representing circular orbits). Predictions generated from such an orrery would have almost certainly misled the scientist. Not every orrery is fit to be true: only those that represent adequately what the world is like instead of how we might wish the world to be. This idea leads to the justification of the orrery. By proper construction and calibration methods and by relying on adequate astronomical observations, an orrery embedding the correct kinematical relationships could be built. It is the same for the theory of trajectories used by the first scientist: by the use of calculation methods that were already well established, the prediction was adequate and thus justified.

According to the functionalist account of knowledge, these functional implementations of belief, truth and justification by the orrery and its relations to the scientist should be sufficient to consider its use as a true justified (material) belief, that is, as subjective thing knowledge. 


\title{
V. CONCLUSION
}

If my account is successful, one can extend Baird's notion of thing knowledge beyond the scope of objective scientific knowledge and account for individual scientists' use of such thing knowledge. Baird explicitly excludes the possibility of subjective thing knowledge because scientific instruments, such as material models, are not inside-the-brain cognitive states. If my account is right, I have shown that a subjective account of thing knowledge is possible if (and perhaps only if) one accepts a functional conception of knowledge and belief. According to the hypothesis of extended cognition, external-to-the-brain cognitive states such as beliefs are possible if one accepts that what makes something genuinely cognitive is not that it is implemented in one's brain but that it plays the relevant role for someone's cognition. If one finds that a material model is used in accord with the functional conception of a belief for a given cognitive system, and that this model also complies with a functional notion of truth and justification, one has a genuine case of subjective thing knowledge.

This extension of Davis Baird's thing knowledge aims to enrich his account of the embodiment of knowledge by scientific instruments by showing how material models can be used as knowledge by some individual scientist instead of solely by a scientific community. Although in this paper I restricted myself to argue for the possibility of subjective thing knowledge, I believe that such use of thing knowledge has played a role in scientific endeavours. Elsewhere I argue that some molecular models in biochemistry were actually used as subjective thing knowledge and that using such a conception of knowledge allows one to better understand the process of discovery by material model-building. ${ }^{9}$

\author{
Mathieu ChaRbonneau \\ Département de Philosophie \\ Université de Montréal \\ 2910, boul. Édouard-Montpetit \\ Montréal, QC H3T 1J7 \\ mathieu.cote-charbonnea@umontreal.ca
}

9 These are, amongst others, Watson' and Crick's 1953 DNA and nucleobases cardboard and metal plates models and Linus Pauling's 1948 use of paper-model of the $\alpha$-helix and his use of space-filling models in order to solve the structure of collagen. 


\section{REFERENCES}

Baird, Davis. 2002. Thing Knowledge-Function and Truth. Techné 6 (2): 13-27.

Baird, Davis. 2004. Thing Knowledge: A Philosophy of Scientific Instruments. Berkeley: University of California Press.

Carruthers, Peter, Stephen P. Stich, and Michael Siegal, eds. 2002. The cognitive basis of science. Cambridge: Cambridge University Press.

Clark, Andy. 2008. Supersizing the Mind : Embodiment, Action, and Cognitive Extension. Oxford: Oxford University Press.

Clark, Andy, and David J. Chalmers. 1998. The Extended Mind. Analysis 58: 10-23.

Frigg, Roman, and Stephan Hartmann. 2006. Models in Science. In The Stanford Encyclopedia of Philosophy, edited by E. N. Zalta. Stanford: The Metaphysics Research Lab.

Lewis, David. 1970. How to Define Theoretical Terms. Journal of Philosophy 67 (13): 427-46.

Lewis, David. 1972. Psychophysical and Theoretical Identifications. Australasian Journal of Philosophy 50: 249-58.

Meeus, Jean. 1999. Astronomical Algorithms. 2nd ed. Richmond: Willmann-Bell.

Popper, Karl R. 1972. Objective Knowledge: An Evolutionary Approach. Oxford: Oxford University Press.

Shoemaker, Sydney. 1981. Some Varieties of Functionalism. Philosophical Topics $12(I): 83-118$.

Sprevak, Marc. 2009. Extended Cognition and Functionalism. The Journal of Philosophy 106: 503-27. 